\title{
Multi-Jet Production Rates in Deep-Inelastic Muon-Proton Scattering
}

\author{
Carlos W. Salgado* \\ Fermi National Accelerator Laboratory \\ P.O. Box 500, Batavia, Illinois 60510
}

October 1992

Presented at the XXVIth International Conference on High Energy Physics, Dallas, Texas, August 6-12, 1992

*Representing the E665 Collaboration 


\section{Disclaimer}

This report was prepared as an account of work sponsored by an agency of the United States Government. Neither the United States Government nor any agency thereof, nor any of their employees, makes any warranty, express or implied, or assumes any legal liability or responsibility for the accuracy, completeness, or usefulness of any information, apparatus, product, or process disclosed, or represents that its use would not infringe privately owned rights. Reference herein to any specific commercial product, process, or service by trade name, trademark, manufacturer, or otherwise, does not necessarily constitute or imply its endorsement, recommendation, or favoring by the United States Government or any agency thereof. The views and opinions of authors expressed herein do not necessarily state or reflect those of the United States Government or any agency thereof. 


\title{
MULTI-JET PRODUCTION RATES IN \\ DEEP-INELASTIC MUON-PROTON SCATTERING
}

\author{
Carlos W. Salgado* \\ Fermi National Accelerator Laboratory \\ P.O.Box 500 \\ Batavia, IL 60510, U.S.A.
}

\begin{abstract}
Measurements of forward multi-jet production rates in deep-inelastic muonproton scattering are presented. Data were taken with a $490 \mathrm{GeV}$ muon beam incident on a hydrogen target. Jets were defined using the JADE jet finding algorithm. The measured rates are presented as function of $W$, the hadronic center-of-mass energy and the jet resolution parameter, $y_{\text {cut }}$, in energies up to $\mathrm{W}=33 \mathrm{GeV}$. Good agreement is found in comparisons with predictions of the QCD-inspired Lund Monte Carlo models. Non-perturbative QCD production mechanisms, inside the Lund Model, can not reproduce the results for energies greater than $W \simeq 20 \mathrm{GeV}$. Sensitivities of the jet rate measurements to the low $x(x \simeq 0.02)$ gluon content of the nucleon and the evolution of $\alpha_{\text {, }}$ are studied.
\end{abstract}

\section{INTRODUCTION}

Deep-inelastic lepton-nucleon scattering (DIS) has been associated with measurements of nucleon structure functions ${ }^{1}$ and, through them, the parton distributions and the strong coupling constant, $\alpha_{\bullet}{ }^{2}$ Measurements of DIS cross-sections represent one of the cleanest ways to obtain the parton distributions and to measure $\alpha_{\boldsymbol{n}}$. Studies of differential cross-sections observing the final hadronic system produced in DIS, as for example multi-jet production, can also be used for the determination of these quantities. However, these measurements have not acquired the same degree of precision as the structure functions measurements. Measurements of multi-jet production rates, by the E665 experiment at Fermilab ${ }^{3}$, have

-Representing the Fermilab E665 Collaboration been possible for the first time due to the highest muon beam energies available at the Tevatron.

First order $\left(\alpha_{s}\right)$ QCD corrections to the DIS Born cross-section include two new final state processes: gluon bremsstrahlung and photongluon fusion, shown in figure 1 . For a fixed beam energy, the kinematics of the order $\alpha$, final state partonic system are defined by five variables. Two variables describe the electroweak vertex kinematics, $Q^{2}$ : the negative square of the virtual photon four-momentum and $x=Q^{2} / 2 P q$, and three describe the final partonic phase space, $x_{p}=Q^{2} / 2 p q, z=$ $p p_{1} / p q$ and $\phi$ the azimuthal angle of the partonic plane relative to the $\mu$ scattering plane. The particle four-momenta: $P, q, p$ and $p_{1}$ are defined in figure 1 . The fraction of the nucleon momentum carried by the struck parton, $\eta$, is given by $\eta=x / x_{p}$, since $p=\eta P$. The total 


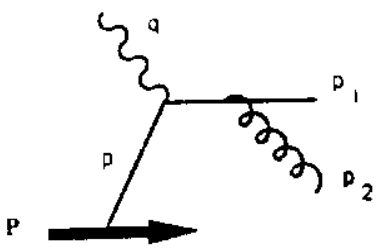

(a)

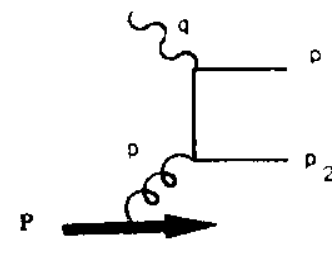

(D)
Figure 1. First order $\alpha_{a}$ corrections to the deepinelastic muon-proton scattering. (a) Gluon bremmstrahlung (b) photon-gluon fusion.

hadronic center-of-mass energy is $W$, related to the previous variables by $W^{2}=(P+q)^{2} \simeq$ $Q^{2}(1-x) / x$.

The production rates for these processes have been calculated in perturbative $\mathrm{QCD}^{4}$. At order $\alpha_{a}$, they are directly proportional to $\alpha_{a}$, in contrast to the more complicated total DIS cross-section expressions.

After fragmentation, given a particular jet finding algorithm and enough center-of-mass energy, these events can be observed as threejet events. They can be described in the virtual photon-proton center-of-mass system as two jets produced by the fragmentation of the final partonic states (mostly going forward with respect to the direction of the incoming virtual photon) and one backward going jet produced by the target remnants. The usual nomenclature for this configuration is $(2+1)$ jets $((1+1)$ for the Born term).

\section{DATA}

The E665 experiment ${ }^{5}$ used a $490 \mathrm{GeV}$ muon beam which struck a $1.15 \mathrm{~m}$ long hydrogen target. Charged particles reconstructed in the tracking system and fitted to the primary vertex and neutral particles reconstructed in the electromagnetic calorimeter are used. The tracking system is essentially in the forward direction (data from the streamer chamber detector that surrounded the target region are

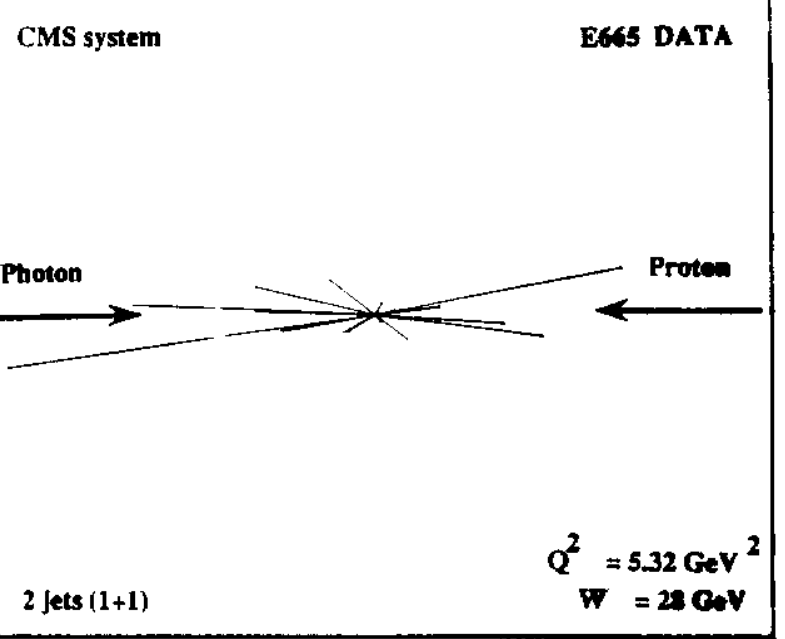

(a)

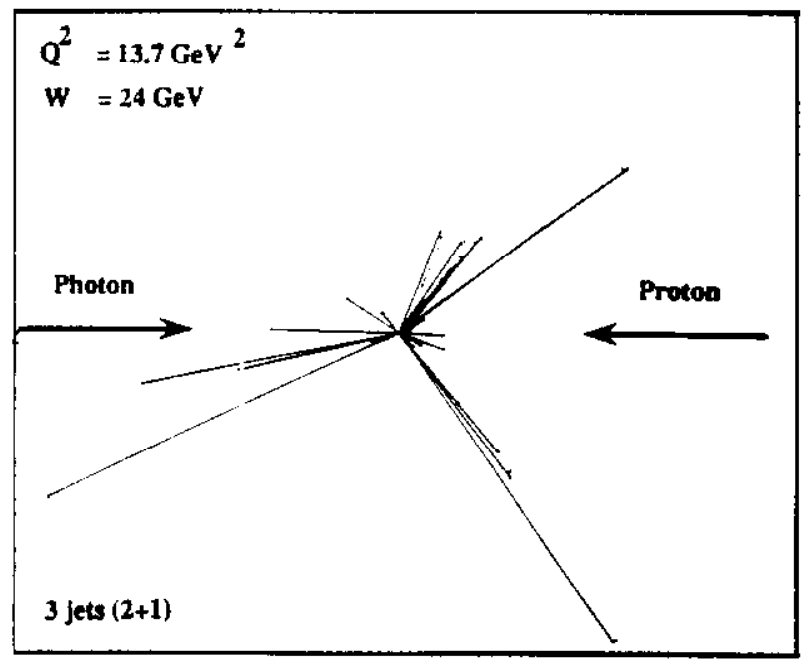

(b)

Figure 2. E665 observed events boosted to the virtual photon-proton center-of-mass frame and projected into the hadronic plane: (a) a $(1+1)$-jets event, (b) a $(2+1)$-jets event. 
not included in this analysis). The event sample is defined by appling the following kinematical cuts: $Q^{2}>4.0(\mathrm{GeV} / \mathrm{c})^{2}, \nu>40 \mathrm{GeV}$, $x>0.003$ and $0.05<\nu / E_{\text {Beam }}<0.95$, where $\nu$ is the energy of the virtual photon in the lab frame. Events from coherent photon bremsstrahlung were identified as such if $\nu>200 \mathrm{GeV}$ and $E_{C a l} / \nu>0.035$ and removed from the data sample. Only particles going forward in the virtual photon-proton center-of-mass system were considered. More details on the analysis can be found in reference [3].

Figure 2 shows two observed E665 events boosted into the virtual photon-proton center of mass system and projected onto the hadronic plane (the plane containing the virtual photon and in which the sum of the squared transverse momenta of the hadrons is maximized). The line segments are proportional to the particle momenta and neutral and charged particles are shown. Electronic and streamer chamber detector information is included. Figure $2 \mathrm{a}$ shows the expected topology of a $(1+1)$ jet event and figure $2 b$ shows a $(2+1)$ jet event, with the backward jet in the direction of the proton and the two forward jets in the direction of the photon. A quatitative jet analysis, such as the measurement of $n$-jet rates, needs a jet "counting" algorithm and corrections for acceptance and detector efficiencies.

\section{JET RATES MEASUREMENTS}

To define the number of jets in an event we use the JADE jet finding algorithm ${ }^{6}$. The JADE algorithm was designed to resemble the technique use in perturbative QCD calculations to overcome soft and colinear singularities. In the experimental implementation of the algorithm, a test variable, $y_{i j}=$ $2 E_{i} E_{j}\left(1-\cos \theta_{i j}\right) /(\epsilon W)^{2}$ is calculated for each pair of hadrons, $i$ and $j . E_{i, j}$ are the parti- cle energies, $\theta_{i j}$ the angle between them and $\epsilon$ an efficiency factor applied to $W$ defining the energy scale. All quantities are calculated in the virtual photon-proton center-of-mass system. The minimum $y_{i j}$ is compared to the jet resolution parameter, $y_{\text {cut }}$. If $y_{i j}^{m i n}<y_{\text {cut }}$ the two particles four-momenta are added to form a new particle $\mathrm{k}$, such that $p_{k}^{\mu}=p_{i}^{\mu}+p_{j}^{\mu}$. The procedure is repeated until $y_{i j}^{\text {min }}$ is larger or equal to $y_{\text {cut }}$. The resulting combined particles are called jets. All charged particles are assumed to be pions and all neutral particles to be photons. The forward n-jet rates are the ratios between the number of events with forward $\mathrm{n}$-jets and the total number of events. We found that using $\epsilon=0.5$ when applying the algorithm to the raw data minimized the detector efficiency and acceptance corrections. The final results were corrected for this choice of scale and are presented for $\epsilon=1.0$.

A GEANT ${ }^{7}$-based Monte Carlo simulation of our detector was used to correct the data distributions for geometrical acceptance, reconstruction efficiency and resolution. The Lund Monte Carlo (LEPTO 5.2 and JETSET $6.3)^{8}$ was used as the physics generator. Both versions of the Lund generator, matrix elements (ME) and parton showers (PS), were able to reproduce many aspects of the data, including the uncorrected jet rates, with similarly good accurancy. The data distributions were corrected bin by bin using:

$$
R_{n}^{\text {corrected }}\left(y_{\text {cut }}, W\right)=\frac{R_{n}^{M C \text { true }}}{R_{n}^{M C \text { recon }}} R_{n}^{\text {dale }}\left(y_{\text {cut }}, W\right)
$$

where $R_{n}^{M C}$ true was obtained applying the JADE algorithm to the primary forward hadrons generated by the Monte Carlo and $R_{n}^{M C}$ recon was obtained from Monte Carlo generated events subjected to the identical analysis as to the data. 


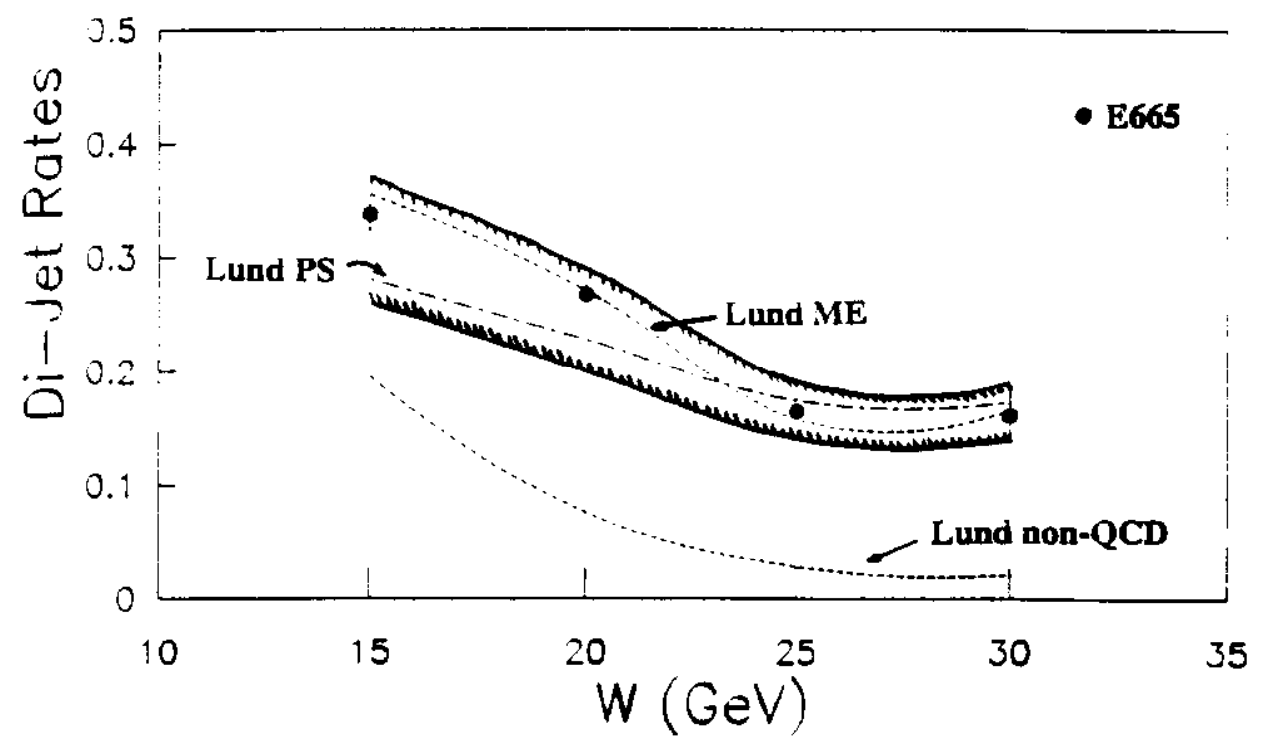

Figure 3. Forward di-jet rates versus $W$, for $y_{c s t}=0.04$. Different Lund model predictions are also shown.

We estimated the systematic uncertainties in the jet rates due to the event and particle selection criteria to be less than \pm 0.01 , and that due to our ability to model the acceptance and efficiency of the apparatus to also be less than \pm 0.01 . We varied the initial choice of energy scale in the definition of $y_{i j}$ from values of $\epsilon$ between 0.3 and 1 , and found that the final corrected jet rates values were very stable, at the \pm 0.015 level. The most important uncertanty came from the physics generator used within the Monte Carlo. We estimated this uncertainty by comparing the corrected rates using the ME and the PS options of the Lund Model. The differences in the resultant rates were $Q^{2}$ dependent but were always less than 0.04 . The combined systematic uncertanties were estimated adding the model dependent uncertainties to the sum in quadrature of all the others. This total systematic uncertainty is always less than 0.06 .

Figure 3 shows the forward di-jet rates versus $\mathrm{W}$ for a value of $y_{\text {cut }}=0.04$. Also shown are the predictions of the Lund ME and PS Monte Carlos. Default values for the parameters in both Lund options and Morfin \& Tung-LO parton distributions ${ }^{2}$ were used. Good agreement is observed with the QCD based models, but the naive parton model based Lund Monte Carlo ("non-QCD") can not explain the data. Error bars represent binomial statistical errors and the marked region, the estimated systematic uncertanties.

\section{QCD ANALYSIS}

QCD information from the hadronic system might be extracted if hadron-parton duality can be established; in other words, if the hadronic jet rates measure the underlying partonic jet rates predicted by perturbative QCD. Figure 4 shows the corrected forward hadronic jet rates compared to the forward partonic jet rates as simulated by the ME Lund Monte Carlo (obtained by applying the JADE finding algorithm to the partons generated by the Lund Monte Carlo). They agree at the $20 \%$ level. The next step will be to find the relation between the forward produced rates and the $(2+1)$ jet rates calculated by perturbative QCD. Different jet finding algorithms might also be explored to minimize these corrections. We are still working to disentangle these effects.

The goal is to obtain information about the gluon distribution function, $\mathrm{G}\left(\eta, Q^{2}\right)$ and the 

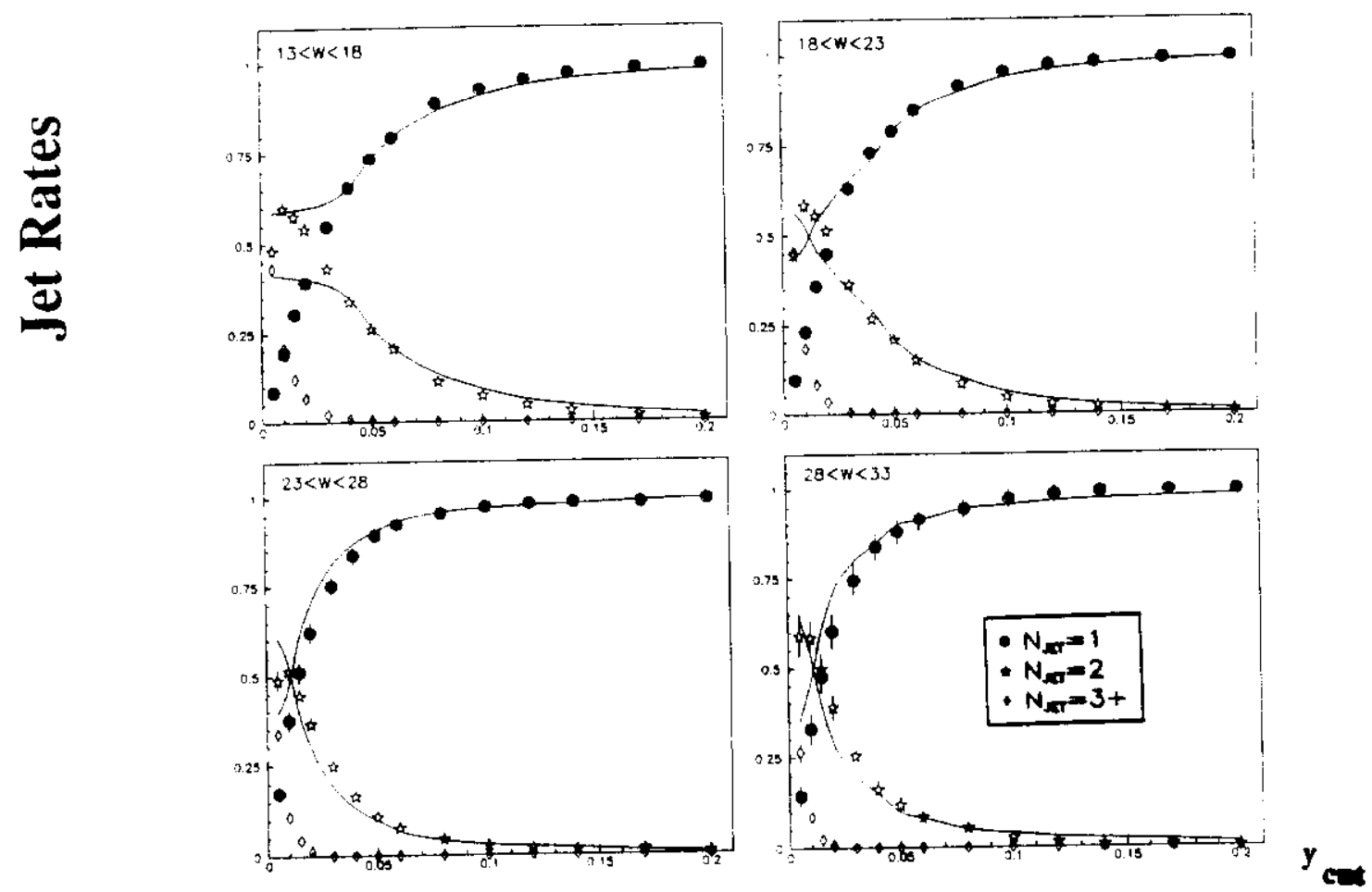

Figure 4. Forward jet rates versus $y_{c s t}$ in four $W$ bins. The solid lines are forward partonic jet rates from the Lund ME Monte Carlo simulation.

strong coupling constant, $\alpha_{\mathbf{s}}$. This information can be obtained by comparing data and perturbative QCD predictions. I will present here our first attempts to estimate sensitivity of the jet rates to $\mathrm{G}\left(\eta, Q^{2}\right)$ and $\alpha_{3}$. To fix the final partonic state phase space, we chose to fix $x=0.02$ and $y_{\text {cut }}=0.04$ (limits of the phase space integration). If we assume that all parton distributions ${ }^{9}$ and the mass of the quarks are known, the only unknown quantity in the $(2+1)$ jet rates is $\alpha_{0}$.

Figure $5 \mathrm{~b}$ shows predictions for the $(2+1)$ jet rates from the ME Lund Monte Carlo model at the partonic level, for fixed (at $Q^{2}=7 \mathrm{GeV}^{2}$ ) and running $\alpha_{.}$. The Lund Monte Carlo predicts, in our energy regime, a maximum difference of rates between $\alpha_{\&}$ fixed and running, of the order of 0.02 . As explained before, the uncertainties associated with our present measurements are of the order of 0.06 .

The gluon distribution function enters the $(2+1)$ jet rates through an integral of the form, $\int_{x}^{1} G\left(x / x_{p}, Q^{2}\right) d x_{p}$. At our values of $x$ the photon-gluon fusion process is expected to have a big contribution to the $(2+1)$ jet crosssection, thus we expect to be sensitive to the form of $\mathrm{G}\left(\eta, Q^{2}\right)$.

To study the sensitivity of the jet rates to the gluon distribution, we chose the form, $\eta G\left(\eta, Q^{2}\right)=A_{0} \eta^{-A_{1}}(1-\eta)^{A_{2}}$ and studied the jet rates sensitivity to changes to the value of $A_{1}$. We keep $A_{2}$ fixed (from LO Morfin \&Tung distributions ${ }^{9}$ ) and change $A_{0}$ to mantain the sum over the gluon momenta constant when changing $A_{1}$. Figure 5a shows the partonic $(2+1)$ jet rates obtained from the Lund ME Monte Carlo for three values of $A_{1} ; \mathbf{0 . 0}, 0.4$ and 0.7. The absolute difference between the rates is of the order of 0.09 . Comparing to our present experimental uncertainty (of the order of 0.06), we expect to be sensitive to values of $A_{1}$ of the order of $\Delta A_{1}=0.7$.

\section{CONCLUSIONS}

Measurements of forward multi-jet production rates have been made in the range $13 \leq$ $W \leq 33 \mathrm{GeV}$ using the JADE jet finding al- 


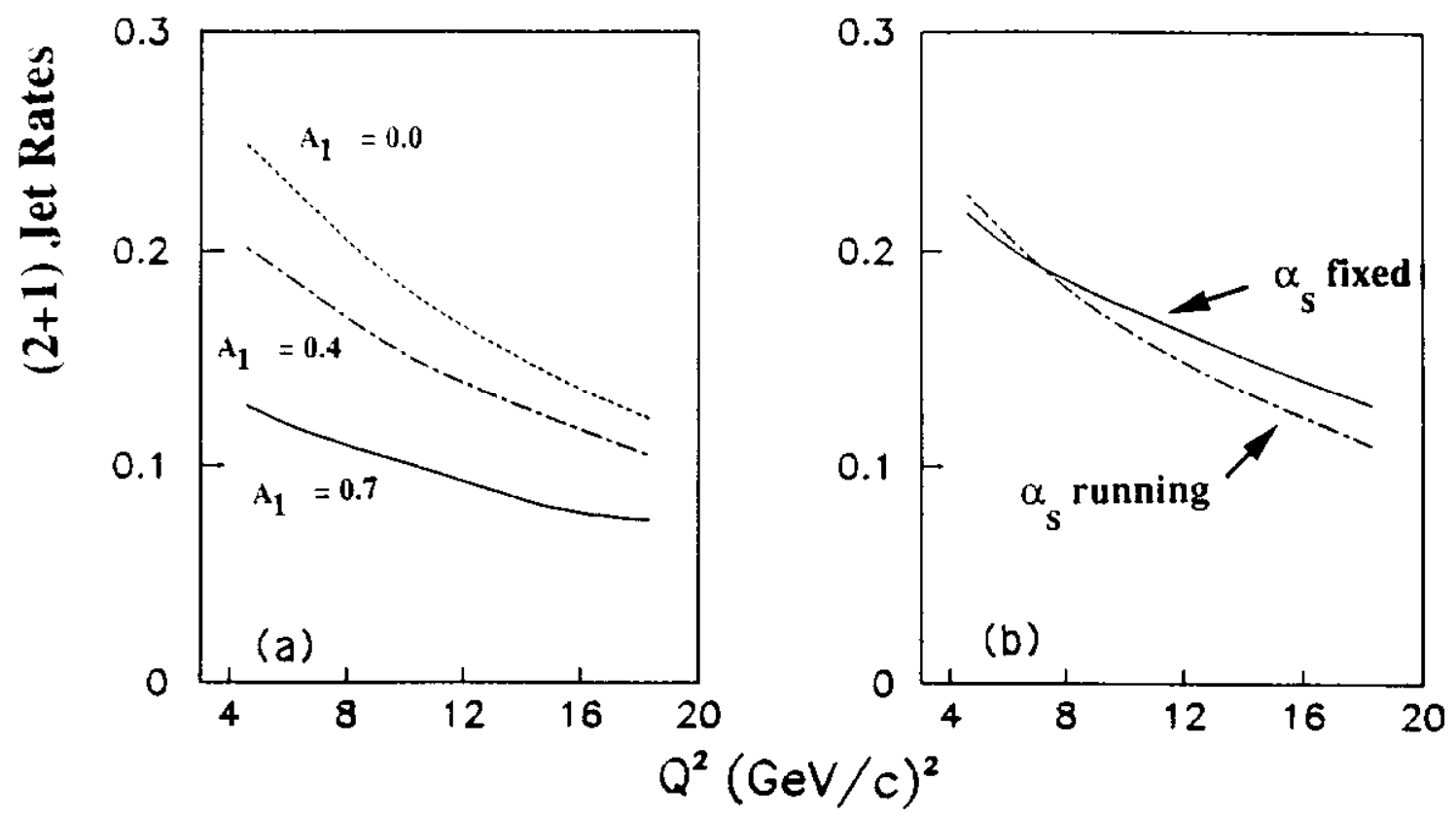

Figure 5. Lund ME Monte Carlo partonic $(2+1)$ jet rates versus $Q^{2},(a)$ for three different choicea of the gluon distribution, and (b) for $\alpha$, fixed (at $Q^{2}=7 G e V^{2}$ ) and running.

gorithm.

Comparisons, at the hadron level, to $\mathrm{ME}$ and pS Lund Model predictions are in good agreement with the data, whereas the predictions of the bare parton model (non-QCD) Lund Monte Carlo are excluded, at least for energies greater than $W \simeq 20 \mathrm{GeV}$. A decrease in the resolved di-jet rates is observed as a function of $W$, in qualitative agreement with perturbative QCD.

Preliminary results from the QCD analysis indicate that the use of the jet rates measurements to determine the running of $\alpha_{0}$ is not possible, at this time, due to the size of our statistical and systematic uncertainties. On the other hand, these measurements can be used to constraint the gluon distribution at low values of $x(x \simeq 0.02)$.

\section{REFERENCES}

1. S.R. Mishra and F. Sciulli, Annu. Rev. Nucl. Part. Sci. 38, 259 (1989).

2. Proceedings of the Workshop on Hadron Structure Functions and Parton Distributions, edited by D. Geesaman, J. Morfin,
C. Sazama and W. K. Tung, World Scientific, Singapore, (1990).

3. M.R. Adams, et al., Phys.Rev. Lett. 69, 1026 (1992).

4. T. Brodkorb, J.G. Körner, E. Mirkes and G.A. Schuler, Z.Phys. C 44, 415 (1989); D. Graudenz, Phys. Lett. B 256, 518 (1991).

5. M.R. Adams, et al., Nucl. Inst. and Meth. A291, 533 (1990).

6. W. Bartel, et al., Z. Phys. C 33, 23 (1986).

7. R. Brun, et al., CERN-DD/EE/84-1 (1987).

8. G. Ingelman, in The Lund Monte Carlo for Lepto-Nucleon Scattering - LEPTO, Version 5.2, unpublished Program Manual..

9. J.G. Morfin and W.K. Tung, Z. Phys. C 52, 13 (1991). 